\title{
Nano-surgery at the leukocyte-endothelial docking site
}

\author{
Christoph Riethmuller • Ines Nasdala • \\ Dietmar Vestweber
}

Received: 17 October 2007 / Accepted: 20 November 2007 /Published online: 19 December 2007

(C) Springer-Verlag 2007

\begin{abstract}
The endothelium has an important role in controlling the extravasation of leukocytes from blood to tissues. Endothelial permeability for leukocytes is influenced by transmembrane proteins that control interendothelial adhesion, as well as steps of the leukocyte transmigration process. In a cascade consisting of leukocyte rolling, adhesion, firm adhesion, and diapedesis, a new step was recently introduced, the formation of a docking structure or "transmigratory cup." Both terms describe a structure formed by endothelial pseudopods embracing the leukocyte. It has been found associated with both para- and transcellular diapedesis. The aim of this study was to characterize the leukocyte-endothelial contact area in terms of morphology and cell mechanics to investigate how the endothelial cytoskeleton reorganizes to engulf the leukocyte. We used atomic force microscopy (AFM) to selectively remove the leukocyte and then analyze the underlying cell at this specific spot. Firmly attached leukocytes could be removed by AFM nanomanipulation. In few cases, this exposed 8-12 $\mu \mathrm{m}$ wide and $1 \mu \mathrm{m}$ deep footprints, representing the cup-like docking structure. Some of them were located near endothelial cell junctions. The interaction area did not exhibit significant alterations neither morphologically nor mechanically as compared to the surrounding cell surface. In conclusion, the endothelial invagination is formed without a net depolymerization of
\end{abstract}

\footnotetext{
C. Riethmuller $(\triangle)$

Institute of Physiology II, University of Münster,

Robert-Koch-Strasse 27 b,

48149 Münster, Germany

e-mail: chrth@uni-muenster.de

I. Nasdala $\cdot$ D. Vestweber

Max-Planck Institute of Molecular Biomedicine,

Münster, Germany
}

f-actin, as endothelial softening at the site of adhesion does not seem to be involved. Moreover, there were no cases of phagocytotic engulfment, but instead the formation of a transmigratory channel could be observed.

Keywords Umbilical vein - Vascular function . Vascular permeability · Neutrophil · Transcytosis . Endothelium · Endothelial cell

\section{Introduction}

Evasion of leukocytes from the blood stream into the tissue is crucial for host defense. On this route, immune cells have to pass the endothelial barrier. Leukocyte extravasation is a multistep-process that starts with tethering, continues with leukocyte rolling and firm adhesion, and finally, results in diapedesis, the actual transmigration step [14, 17, 25]. Groups of transmembrane proteins have been identified responsible for different phases, namely the selectins for tethering and rolling [26], and integrins for firm adhesion of leukocytes to the endothelial intercellular adhesion molecule-1 (ICAM-1) and vascular cell adhesion molecule-1 [3, 30]. Further passage through the endothelial cell layer is promoted by platelet/endothelial cell adhesion molecule-1 and CD99 [4, 27]. Diapedesis is generally assumed to take place in a paracellular manner (between the cells), but also a transcellular pathway (through an endothelial cell) has recently been demonstrated [9]. Up to now, it is not known what determines leukocytes to use one or the other route.

Recently, contacts between leukocytes and the apical endothelial cell surface were described as docking structure [3], formed by endothelial cell membrane ruffles or filopodia intimately embedding the leukocyte [5]. At the same time, the endothelial cell invaginates at these sites. 
Because of its shape and function, this structure has also been termed "transmigratory cup" [6]. To represent both morphology and function, we here use the expression "cuplike docking structure" (CLDS). ICAM-1 is highly enriched at leukocyte-endothelial contact sites and is essential in steering the diapedesis process $[1-3,13]$.

The rapid change of cell shape in the course of cup formation cannot be driven without a drastic local rearrangement of the cytoskeleton. It has been hypothesized that the mechanism involves cortical actin remodeling via activation of RhoA [1]. Very recently, RhoG has been assigned a central role via activation through SH3 [24]. However, the transmigratory cup does not seem to induce a preference for transcellular diapedesis, as quantified from colocalization of leukocyte and endothelial junction-marker proteins. The criteria that determine which route a leukocyte prefers are unknown. A technique well suited for the analysis of cellular mechanics is atomic force microscopy (AFM), which has proven to deliver novel information about cytoskeletal arrangements affecting cell stiffness in physiological processes $[7,16]$. Up to now, it was already successfully used to explore the interaction forces between endothelium and immune cell $[8,32]$, and it has been applied to determine mechanical characteristics of leukocytes [21].

In this study, we make use of the mechanical working principle of AFM to obtain morphological hints from the leukocyte-endothelial contact site to get a more detailed view on whether the transmigratory cup drives a certain preference for a para- or transcellular transmigration pathway. We, in this paper, show that CLDSs can be observed after leukocytes have been removed and that they can be located in direct vicinity to an endothelial cell junction that stays intact.

\section{Materials and methods}

\section{Cell isolation and cultivation}

\section{Mouse system}

Cells from the stable line bEnd5 derived from mouse brain endothelium were cultured and passaged as described [18]. For transmigration assays, bEnd5 cells were grown on filter inserts $\left(0.31 \mathrm{~cm}^{2}, 5 \mu \mathrm{m}\right.$ pores Falcon, BD, purchased through VWR, Darmstadt, Germany) and stimulated with TNF $\alpha 19 \mathrm{~h}$ before inoculation with polymorphonuclear leukocytes (PMNs).

\section{Preparation of mouse bone marrow neutrophils}

NMRI mice (Harlan-Winkelmann, Borchen, Germany) were killed, femurs and tibias were cut out, and muscles were removed. Bones were placed in $\mathrm{Ca}^{2+} / \mathrm{Mg}^{2+}$-free Hank's buffered salt solution ( $\mathrm{HBSS}^{-/}$; Invitrogen) to prevent dryout. The ends of the bones were cut, and the bone marrow was flushed into a 50-ml tube with $\mathrm{HBSS}^{-/-}$using a short (5/ 8-in.) 25-G needle and a 2-ml syringe. Larger bone marrow pieces were disaggregated using a plastic pipette and a mesh $(70 \mu \mathrm{m})$. The cell suspension was centrifuged (at $300 \times g$, $10 \mathrm{~min}$ ), and the pellet was resuspended in $1 \mathrm{ml}$ of $\mathrm{HBSS}^{--}$. The cell suspension was then added on top of a 10-ml tube containing $4 \mathrm{ml}$ of Histopaque 1119 and $4 \mathrm{ml}$ of Histopaque 1077 (Sigma-Aldrich, Steinheim, Germany). After centrifugation for $30 \mathrm{~min}$ at $700 \times \mathrm{g}$ (brake off), the polymorphonuclear cell (PMN)-containing cell layer was collected and washed two times with $\mathrm{HBSS}^{-/}$.

\section{Human system}

Endothelial cells (human umbilical vein endothelial cell, HUVEC) were isolated from umbilical veins as described [11], seeded on glass cover slips coated with gelatine and cultivated in DMEM with $0.1 \%$ penicillin/streptomycin. Cells were stimulated with $5 \mathrm{nM} \mathrm{TNF} \alpha 19 \mathrm{~h}$ before experiments.

Neutrophils were isolated from fresh donor blood according to a standard protocol using centrifugation in a ficoll gradient. Briefly, $10 \mathrm{ml}$ heparinized blood were diluted with $10 \mathrm{ml} \mathrm{Ca}{ }^{2+} / \mathrm{Mg}^{2+}$-free phosphate-buffered saline $\left(\mathrm{PBS}^{-/-}\right)$, put on a ficoll solution $(1.077 \mathrm{~g} / \mathrm{ml}$, Biochrom, Berlin, Germany) and centrifuged at $900 \times g$ (without brake). The erythrocyte/granulocyte pellet was resuspended in $\mathrm{PBS}^{-/-}$, mixed 1:1 with RPMI1640 medium, and added with dextran solution $0.4 \%$ final concentration and sedimented in the incubator at $37^{\circ} \mathrm{C}$, and the upper phase was centrifuged and washed.

\section{Immunofluorescence}

Samples were fixed in 4\% paraformaldehyde for $1 \mathrm{~h}$ at room temperature, washed with PBS, permeabilized with $0.1 \%$ Triton and $1 \%$ bovine serum albumin (BSA), and incubated with the antibodies at $4{ }^{\circ} \mathrm{C}$ overnight in a dark humid chamber. The cells were washed three times for $10 \mathrm{~min}$ in PBS and mounted with Crystalmount. The samples were analyzed with a fluorescence microscope (Axiovert 200, Zeiss, Oberkochen, Germany) and a digital camera CoolsnapHQ (Visitron, München, Germany).

\section{Antibodies}

Antibodies that include anti-human ICAM-1-FITC, antihuman CD66 PE, and anti-mouse Ly6G were from Pharmingen (purchased through BD, Heidelberg, Germany); anti-mouse $\mathrm{YN} 1 / 1.7$ rat IgG was purified from hybridoma cells as described [23]. 
Atomic Force Microscopy (AFM)

With $A F M$, all samples were fixed by addition of glutardialdehyde directly into the assay vial $(0.5 \%$ final concentration, $37^{\circ} \mathrm{C}, 45 \mathrm{~min}$; for elasticity measurements, only $0.05 \%$ ) and washed vigorously with 4-2-hydroxyethyl-1-piperazineethanesulfonic acid (HEPES) buffer three times. Cell imaging was performed in standard contact mode as described before [22]. Briefly, AFM measurements of HUVEC were performed in HEPESbuffered solution ( $\mathrm{pH}$ 7.4) using a Multimode with Nanoscope III controller under software version $5.12 \mathrm{~b} 48$ (VEECO, Mannheim, Germany). Silicon-nitride tips on V-shaped gold-coated cantilevers were used $(0.03 \mathrm{~N} / \mathrm{m}$, MLCT, purchased through VEECO, except for Fig. 6: $0.06 \mathrm{~N} / \mathrm{m} \mathrm{CSC} 17 \mathrm{Cr} / \mathrm{Au}$ purchased through Anfatec, Oelsnitz, Germany). Nanomanipulation was performed using the second-softest tip at very low force for imaging (setpoint below $0.5 \mathrm{~V}$ ); for removing the leukocyte, both the force and scan speed were increased by at least an order of magnitude until the success was detectable in the raw image. To obtain the Young's modulus (YM) describing sample stiffness, arrays of $64 \times 64$ force-distance curves were recorded by the "force volume" option implemented in the software, where the deflection of the cantilever (in nanometer) is taken as a function of the piezo elongation ( $z$ distance in nanometer). Piezo travel speed was kept below $3 \mu \mathrm{m} / \mathrm{s}$. Reconstruction of height maps and YM from the raw data were processed by a program applying the Hertzian model of elasticity as described earlier [20].

\section{Results}

This study was designed to explore the specific part of the apical endothelial membrane that has been in intimate contact with the invading leukocyte. The samples were chosen to be prepared along routine protocols for the assessment of transmigration capacity in a Boyden chamber-like assay with endothelium grown on filter membranes. First, optimal leukocyte counts and interaction time intervals were determined to optimize the assay for AFM requirements. Leukocyte density was controlled by immunohistochemistry with antibodies against typical marker proteins (Fig. 1a). YNI-1 was used to stain bEND5 endothelial cells (not shown) and Ly6G marked PMNs. The aim was to obtain one to five leukocytes within the maximum scan size of $(100 \mu \mathrm{m})^{2}$, which was achieved by
Fig. 1 Mouse cell system on permeable filter membranes. Mouse bEnd5 endothelial cells were grown to confluency, stimulated with $\mathrm{TNF} \alpha$, added with bone marrow PMNs for $20 \mathrm{~min}$, and were fixed. a Fluorescence micrograph of neutrophils marked with antibodies against Ly6G. b, c AFM contact mode images under buffer conditions of $\mathbf{b}$ endothelial cells without and $\mathbf{c}$ with a single neutrohilic leukocyte. The AFM images are color coded: Highest areas of an object are shown in white. Different surface morphologies can be recognized: Whereas the leukocyte has many knot-like protrusions, the endothelium exhibits rather long parallel filaments. Scale Bars are $10 \mu \mathrm{m}$

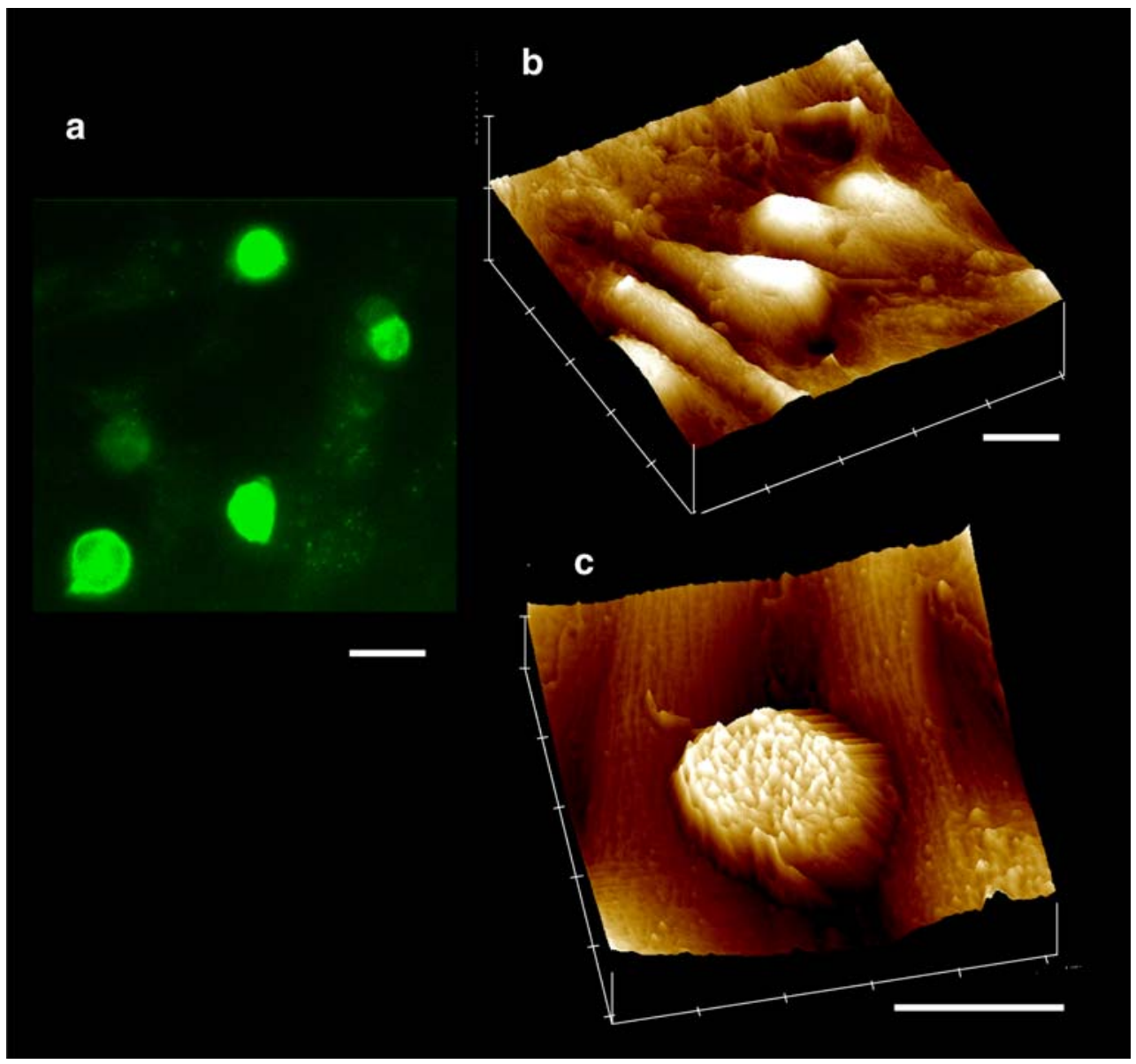


inoculating 100,000 leukocytes per well for $20 \mathrm{~min}$ before fixation (Fig. 1a). Typical AFM data are shown for bEnd5 (Fig. 1b,c). Colors in the AFM images code for the sample height: the higher an area, the brighter its color. The cell line bEnd5, which has been derived from mouse brain endothelial cells, shows a moderate spindle-like appearance with rather flat cell bodies as is common for well-adherent cells. The microstructure allows to recognize quite a number of filopodia, which miss a clear organization and are reminiscent of membrane blebbing or cell debris (Fig. 1b). This phenomenon is not very prominent in light microscopy images but is often observed under the AFM in our lab on stable endothelial cell lines like EA.hy926 or GM7373 (unpublished observation). In Fig. 1c, a single leukocyte is shown associated with the endothelial cell carpet. It exhibits a circular shape of typically $10 \mu \mathrm{m}$ diameter. The surface is covered throughout with filopodiaor vili-like structures, one of which, at the upper left part, seems to invade the endothelial cell underneath. Contrastingly, on the endothelia, directionalized long filaments can be seen, most probably resembling actin fibers. Their characteristic appearance makes it easy to distinguish leukocytes from endothelial cells within the AFM images. The AFM data also are in line with fluorescence microscopy data of identical samples as far as leukocyte density is concerned.
To describe the different stages of the invasion process, we performed a series of transmigration experiments, fixing the cells with glutardialdehyde at different time points after addition of the leukocytes. Although the number of leukocytes observable at the apical surface decreased slightly with increasing inoculation time, the degree of heterogeneity was not reduced. Therefore, the experiments were restricted to one inoculation interval $(20 \mathrm{~min})$. In Fig. 2, examples of various stages of the transmigration process as observed by AFM under fluid are shown in three-dimensional images. An overview of a typical area is given in Fig. 2a, when four (circular) leukocytes are located on four (spindle-like) cells. Of note, samples are rinsed vigorously before imaging so that only firmly attached leukocytes are present. One of them - to the right side - has a highly ruffled surface reminiscent of a cauliflower or rose and a considerably larger diameter. This one probably has already begun to invade the cell underneath and was not removable by increased force. Figure $2 b$ shows a specimen in the course of transmigrating a large pore of the filter membrane (here in black). It obviously has already crossed the endothelium as can be deduced from a neighboring leukocyte being partly covered with a thin endothelial lamellipodium. One leukocyte as well fixed in the act of trespassing is shown in Fig. 2c: More than half of it has

Fig. 2 Leukocytes at different stages of invasion as viewed by AFM. a Scenery of four circular leukocytes attached to three + spindle-like bEND5 endothelial cells, the one most to the right side being engulfed by endothelial protrusions, which can better be recognized in $\mathbf{b}$ the zoomed image; the shape of the leukocyte is copied in yellow to the middle of the tableau. c A leukocyte trapped in the process of evading through a large hole in the filter; it has already passed the endothelial layer. d A leukocyte fills a large hole in the filter; in front, unfilled filter holes covered by endothelium can be seen. Scale bars are $10 \mu \mathrm{m}$

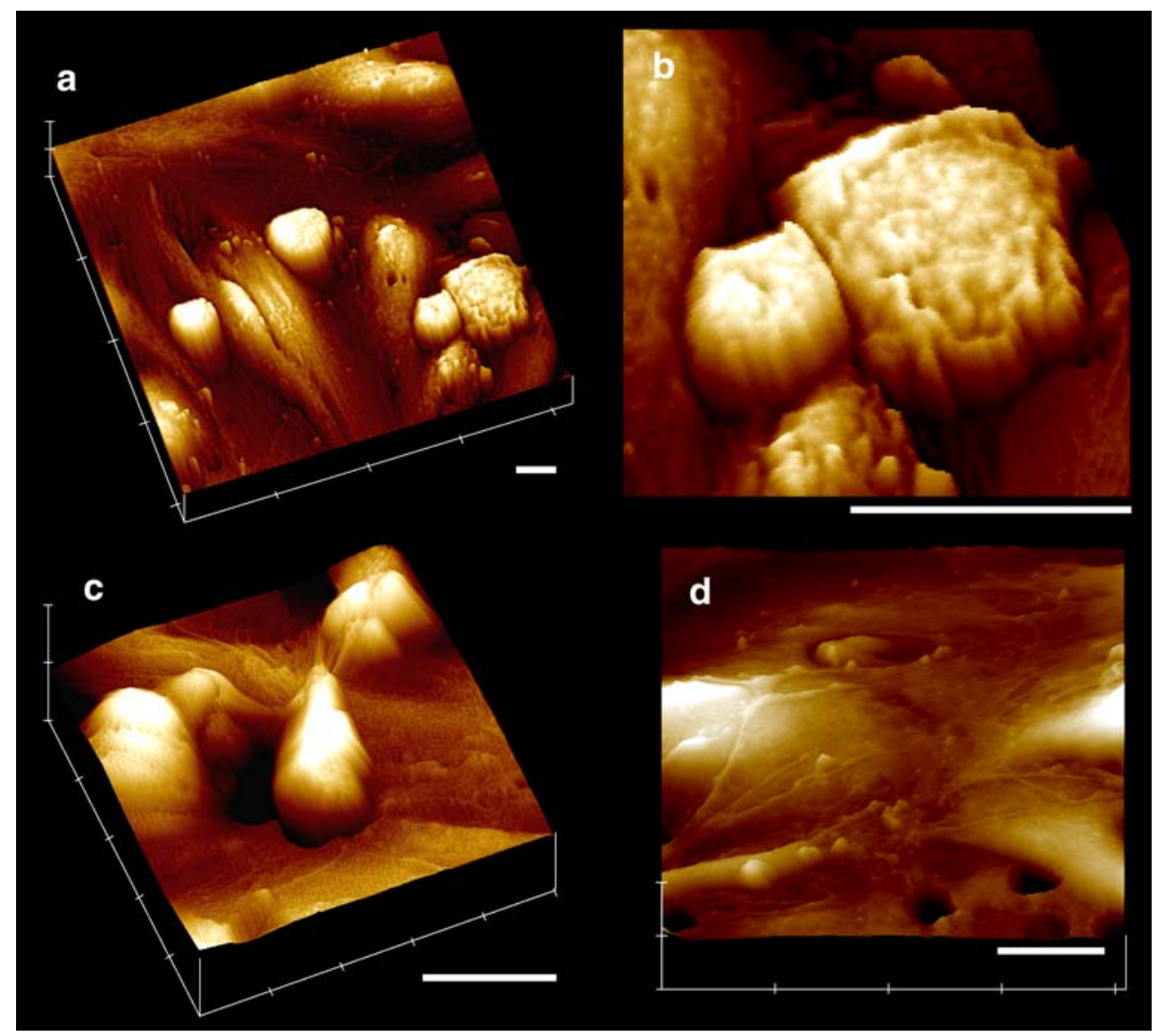


already disappeared beneath an endothelial cell whose actin bundles are spun in parallel, squeezing down the immune cell. In this case, only a segment remains uncovered. Finally, the rear part of a leukocyte can be seen filling a large hole in the membrane (Fig. 2d). Examples of unfilled holes can be seen in front of the image. Nevertheless, these are also covered by endothelial cells, but they appear invaginated because the endothelial cells are so thin and soft that they cannot fully compensate even a minute force load of the AFM tip $(<1 \mathrm{nN})$.

This variety of shapes in mind, the question resurfaces as to when a transmigratory cup is formed and whether it can be rendered accessible to the AFM-sensor tip. As AFM is not only an imaging instrument but also a tool for nanomanipulation, it was, in this study, used to loose the connection between the leukocyte and its engulfing endothelial cell. As described in more detail in the methods section, vertical and lateral forces were tuned up until the leukocyte leaves its binding site (Fig. 3). In Fig. 3a, an image of the leukocyte is presented. The manipulation step-indicated by the sketch in the upper part of the figure - does not yield an image. Afterwards, the identical area is scanned again. Many leukocytes withstand this procedure, but more than 30 could be removed in this way. Independent of interaction time, different classes of leukocytes were observable. (1) Those that were removed by vigorous washing. (2) Of the remaining ones, not all could be imaged by AFM because their attachment was not firm enough. (3) The majority could be imaged and then be removed by nanomanipulation but did not show a CLDS underneath. (4) Very few cases were removable, exhibiting a CLDS in the endothelial cell, and (5) some were not removable without damaging the sample.

One example of the desired class (4) cases is presented in Fig. 3b, clearly exhibiting a (dark) invagination exactly at the position where the leukocyte was formerly attached. This "footprint" obviously is a CLDS. It is not strictly circular, but the dimensions of $8-12 \mu \mathrm{m}$ diameter and up to $1 \mu \mathrm{m}$ depth are reasonable. At the left front part of the footprint, there is a notch-like extension reminiscent of a big toe having been imprinted there. Indeed, when looking at the "before"-image with leukocyte (Fig. 3a), there is a filopodial protrusion at this spot. In this case, the CLDS is located across an endothelial cell junction. The cell border usually is a furrow, which is - depending on the tightness
Fig. 3 Nano-surgery at the docking site. Samples were prepared as in Fig. 2. a AFM contact image of a firmly attached leukocyte exhibiting a protrusion invading the endothelium. In a next run, increased force and high lateral speed were applied to tear out the leukocyte. b Repeated contact imaging of the identical area reveals a CLDS underneath. Here, it is located across a celljunctional ridge. c, d The height profiles along the white lines in the images demonstrate how an $8-\mu \mathrm{m}$ protrusion turns into an 1 $\mu \mathrm{m}$ invagination through distraction of the immune cell

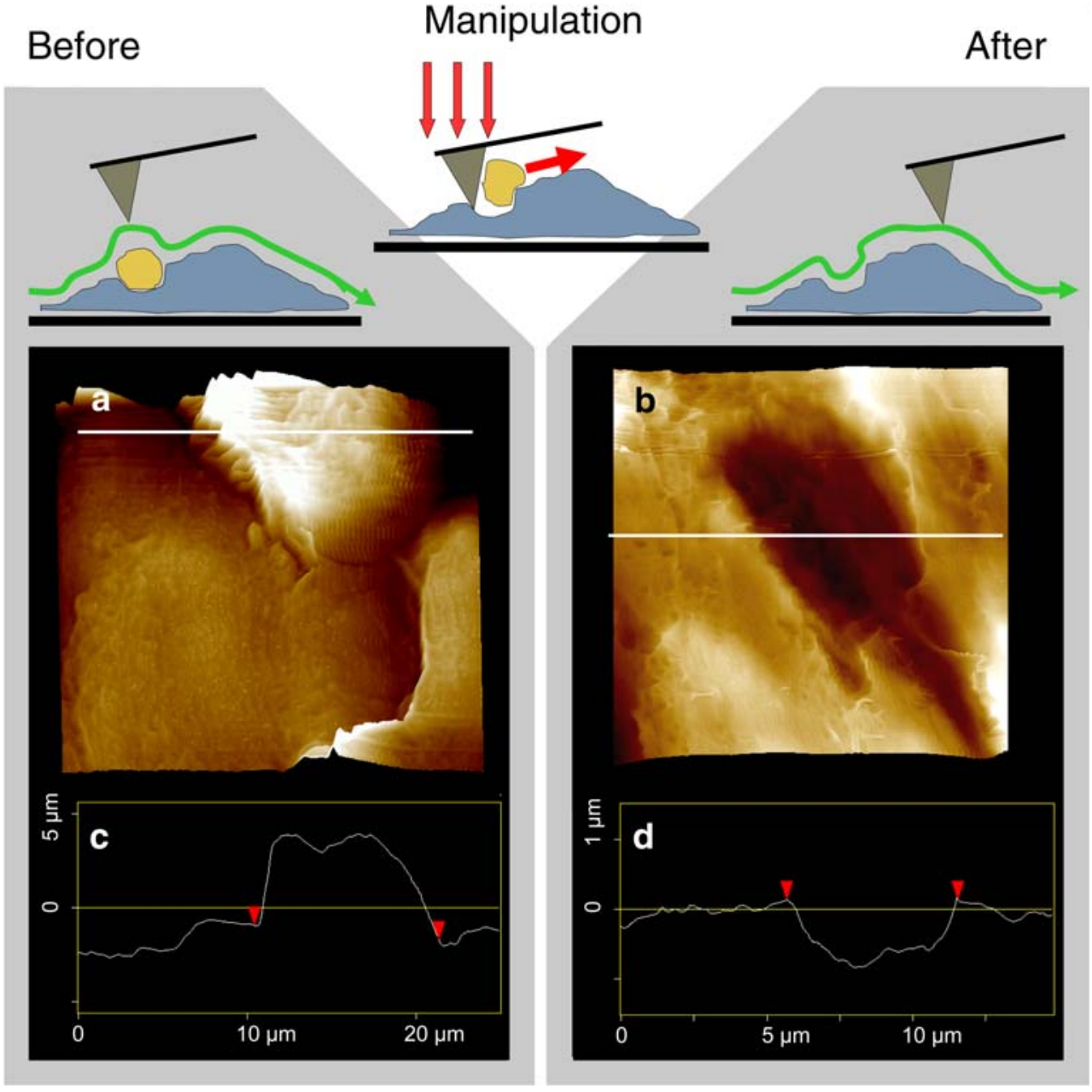


of the junction-flanked by two ridges. Here, after leukocyte removal (Fig. 3b), it forms a furrow outside the CLDS region and mainly a ridge inside, as seen from the height profile (Fig. 3d). We will come back to this point in the section with human cells.

Comparison of the surface ultrastructure did not reveal significant differences between the control and the leukocytedenuded regions. Most importantly, no sites of invadopodia protrusion-which should appear like holes-could be detected. Another question emerges as to the cytoskeletal rearrangements within the endothelial cell. Do the actin filaments depolymerize or retract at the invasion site to form a transmigratory path? If they depolymerize, then the endothelial cell should soften at the prospective entry site as compared to the surrounding area, as the mechanical rigidity of a cell mainly constitutes from actin fibers. Being a mechanical instrument, AFM cannot only image soft samples under buffer conditions but can also analyze mechanical properties. In this study, we performed a force mapping of a leukocyte before (Fig. 4a,c) and of the putative entry site after the nanomanipulation. The upper part of the figure shows the measurement principle: The tip is moved to stitch the sample $64 \times 64$ times, each time
Fig. 4 Mechanical characteristics of the leukocyte-endothelial interface. PMNs were added to bEND5 endothelial cells grown on filter membrane, fixed, and investigated in PBS. a AFM was used as a mechanosensor gently stitching down into the sample in an array of $64 \times 64$ points, yielding 4,096 force distance curves. From these, maps of b and c, $\mathbf{d}$ and e height sample elasticity can be calculated. Note that, in height images, the high regions are given in white, whereas in elasticity maps, bright coloring represents stiff regions. b The clearly elevated leukocyte is d considerably softer (darker) than the surrounding endothelium. After nanomanipulation procedure as in Fig. 3, the repeated force volume analysis of the same spot demonstrates $\mathbf{c}$ the loss of the leukocyte and e a homogeneous elasticity without clear differences at the interfacial area (green circle in $\mathbf{d}$ and $\mathbf{e}$ )
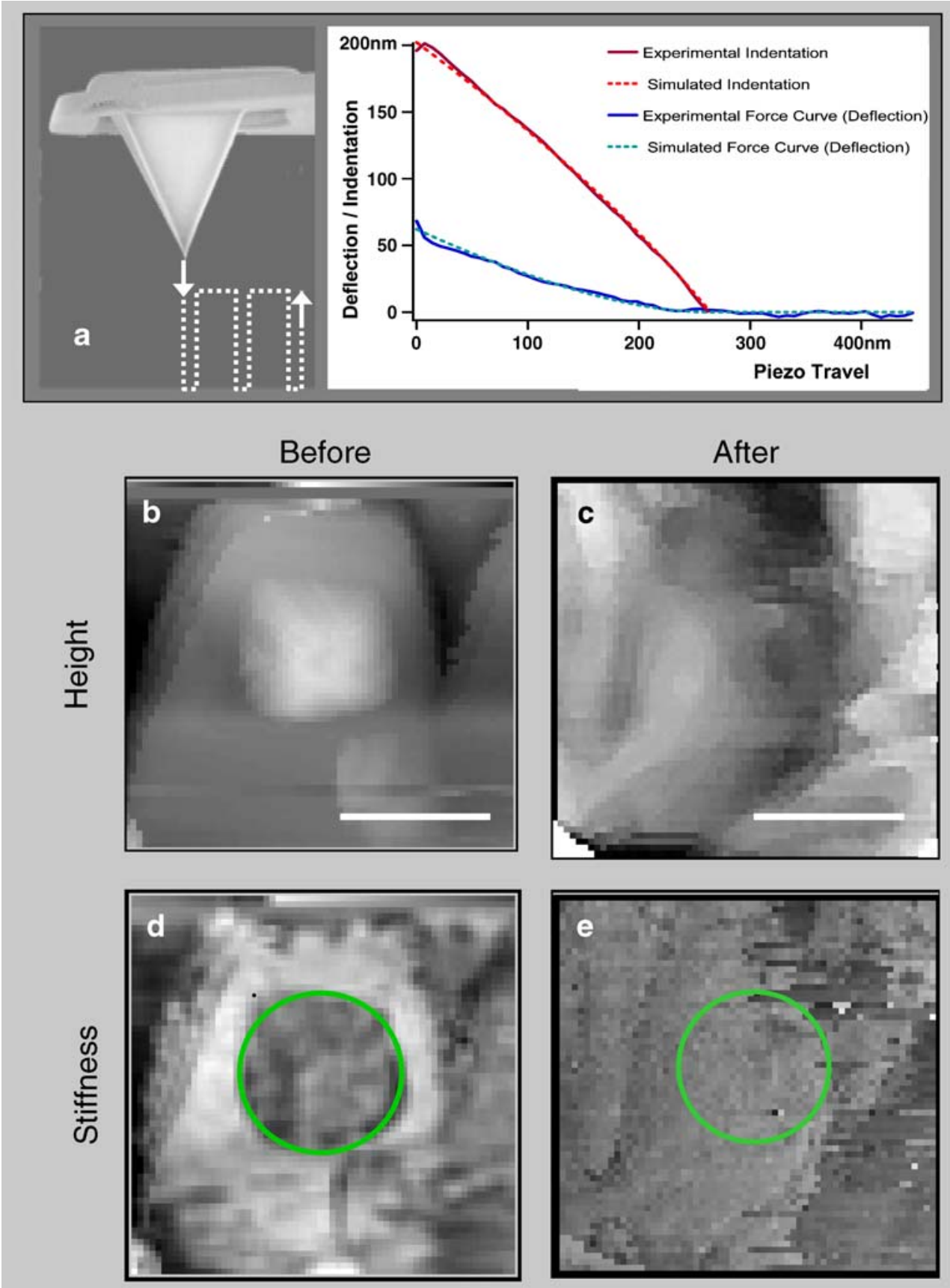
recording a force-distance curve. From these 4,096 curves, maps of sample height (Fig. 4a,b) and stiffness (Fig. 4c,d) can be reconstructed. The appearance of the leukocyte (Fig. 4a,c) is highly disturbed by the AFM cantilever so that only the central region within the green circle is data from the tip. The height images are analogous to the contact mode images shown before. The stiffness maps are differently coded: Stiff regions are shown in white and soft regions in dark. Figure $4 \mathrm{c}$ shows a remarkably softer (darker) appearance of the leukocyte as compared to its endothelial surroundings. This should not be overinterpreted, as the geometry of the leukocyte is not as flat as in endothelial cells. But after removal of the leukocyte, the former contact region that constitutes a putative entry channel (green circle) is not remarkably darker than the average endothelial cell. Although absolute numbers for YM are in a fairly high range of $50 \mathrm{kPa}$ because of fixation, a relative difference might have been detectable if drastic enough. Therefore, a local softening cannot be stated from these data, saying that actin-depolymerization, probably, is not taking place to a large extent. Of note, local differences can also be detected in fixed specimen, although their relative change decreases because of an overall increase in stiffness.

Another characteristic feature of diapedesis is ICAM-rich endothelial filopodia grasping for the leukocyte. This ICAMdriven embracement could be interpreted to set the starting point of the actual diapedesis step. However, we did not observe endothelial filopodia in the mouse cellular system, although AFM should be able to detect them. Therefore, we established the nanomanipulation assay also for primary HUVEC cultivated on glass cover slips, the model system that many fluorescence microscopic reports rely on. Upon activation with TNF- $\alpha$ and adding neutrophils freshly isolated from human blood, the picture was quite similar to the mouse system (Fig. 5). ICAM-1 (Fig. 5a) and CD66 (Fig. 5b) were used as markers for the immunohistochemical characterization of the two cell types. The main morphological differences are the smoother and clearer surface of the primary human endothelial cells on the one hand and, on the other, a rather sponge-like net of ridges in the ultrastructure of the human neutrophiles (Fig. 5c), contrasting the rather spotty surface of mouse leukocytes (Fig. 1c).

The nanomanipulation procedure could successfully be applied to the human cell system as well, an example being shown in Fig. 6. Again, the CLDS lie across an endothelial cell junction (Fig. 6a), and after removal of the leukocyte, the CLDS turns out to be separated into halfs at both sides of the cell border (Fig. 6b). The height profiles given in Fig. 6d,e demonstrate the junctional area forming a ridge (marked by an arrow in $b$ and e). In another example, the CLDS again lies on top of a cell junction (Fig. 7a). In this case, endothelial filopodia remain visible, which most probably have been formerly attached to the leukocyte as demonstrated in the three-dimensional view (Fig. 7b). A height profile yields a diameter of 100-200 nm. In Fig. 8 there is one case, where the transmigratory channel was accessible for AFM nanomanipulation. The artificial coloring depicts the glass bottom in deep blue and the cell nuclear regions in red (Fig. 8a). Obviously, the lamellipodium was flat enough to allow a channel-forming leukocyte being detached through the action of the AFM tip. In the three-dimensional view of Fig. 8b, the footprint is better visualized. The height profile (Fig. 8c) along the white line in Fig. 8a clearly shows the horizontal line from $31-35 \mu \mathrm{m}$, representing the glass cover slip. The inset profile highlights the twin pair of cortical actin bundles resembling a more or less intact endothelial cell junction-indicated by the dotted vertical line. Here, a transmigratory tunnel has been formed through the action of the leukocyte, which spans from the luminal to the abluminal side of the endothelial cell.

\section{Discussion}

The nanoscopic resolving power of AFM even in fluid has proven to contribute valuable information about epithelial and endothelial physiology $[15,22]$, and it promises to add new details also to the knowledge of the transmigration process of leukocytes. This study was designed to explore the leukocyte-endothelial docking structure from a micromechanical point of view, combining the imaging, manipulating, and analyzing capabilities of the versatile method AFM.

The experimental assessment of transmigration is complicated because it is a multistep process, and the last step is a quick one (1-2 min). A further complication is based on the fact that two different pathways exist-namely, through the cells or between them [17], options that leukocytes share with fluid [12, 19]. Direct live observation of the transcellular diapedesis has been published before [31]

The data presented in this paper are novel in several respects. The leukocyte-endothelial transmigration has been investigated by AFM in this study for the first time. As we used standard filter membranes, our experiments can be directly compared with standard transmigration assays. In addition, this is the first time that nanomanipulation has been applied in this system. The CLDS and filopodia could be confirmed as stable morphological entities in wet samples by a method principally different from fluorescence microscopy. Moreover, endothelial filopodia grasping for the leukocyte could be observed as well, although only for human endothelial cells.

So far, the use of AFM in this field is sparse and focuses on the detachment forces between immune cell and endothelial cell [32] or on force spectroscopy of single relevant proteins like ICAM-1/2 [28]. A reason might be 

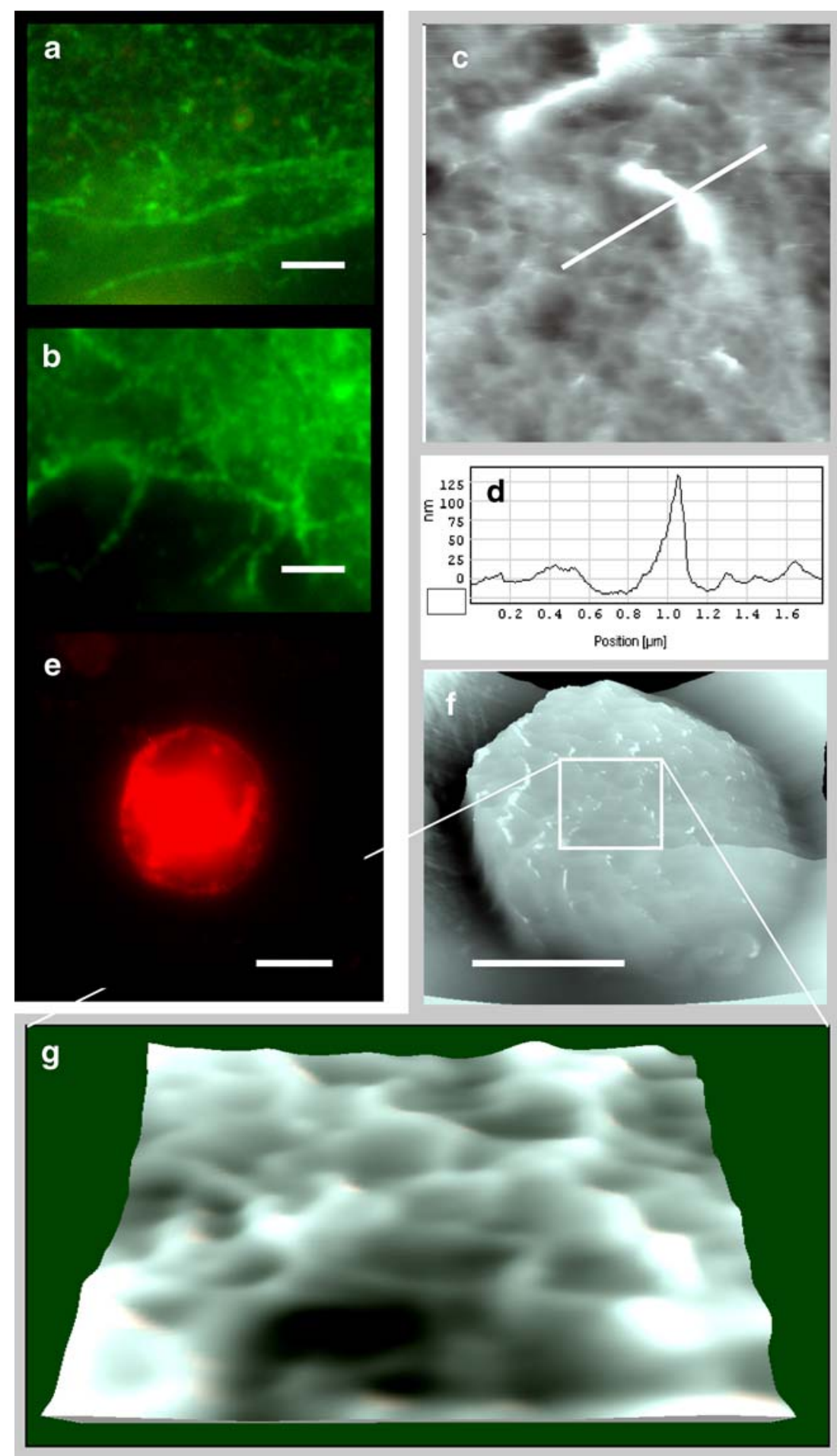

Fig. 5 Human cell system. Freshly isolated blood neutrophils were added to primary human endothelial cells (HUVEC) grown on glass cover slips stimulated with TNF $\alpha$. a, b Fluorescence micrographs of endothelial cells marked with antibodies against ICAM-1. Scale bar is $5 \mu \mathrm{m}$. Finger-like structures can be recognized that do not necessarily coincide with endothelial junctions. c AFM contact image of finger-

the steep slopes around the leukocytes, which are principally inaccessible to the AFM tip and, thus, often result in "blind" coronas around them. Imaging also is often like protrusions on an endothelial cell and $\mathbf{d}$ corresponding height profile. e Fluorescence micrograph of a leukocyte marked with antibodies against CD66. f, g AFM contact image of a neutrophil sitting on endothelium; $\mathbf{g}$ zoom into the top region reveals a spongelike surface

hampered by leukocytes sticking to the AFM tip, thereby, increasing the consumption of AFM tips. One study reports on mechanical characteristics of HUVEC in response to 

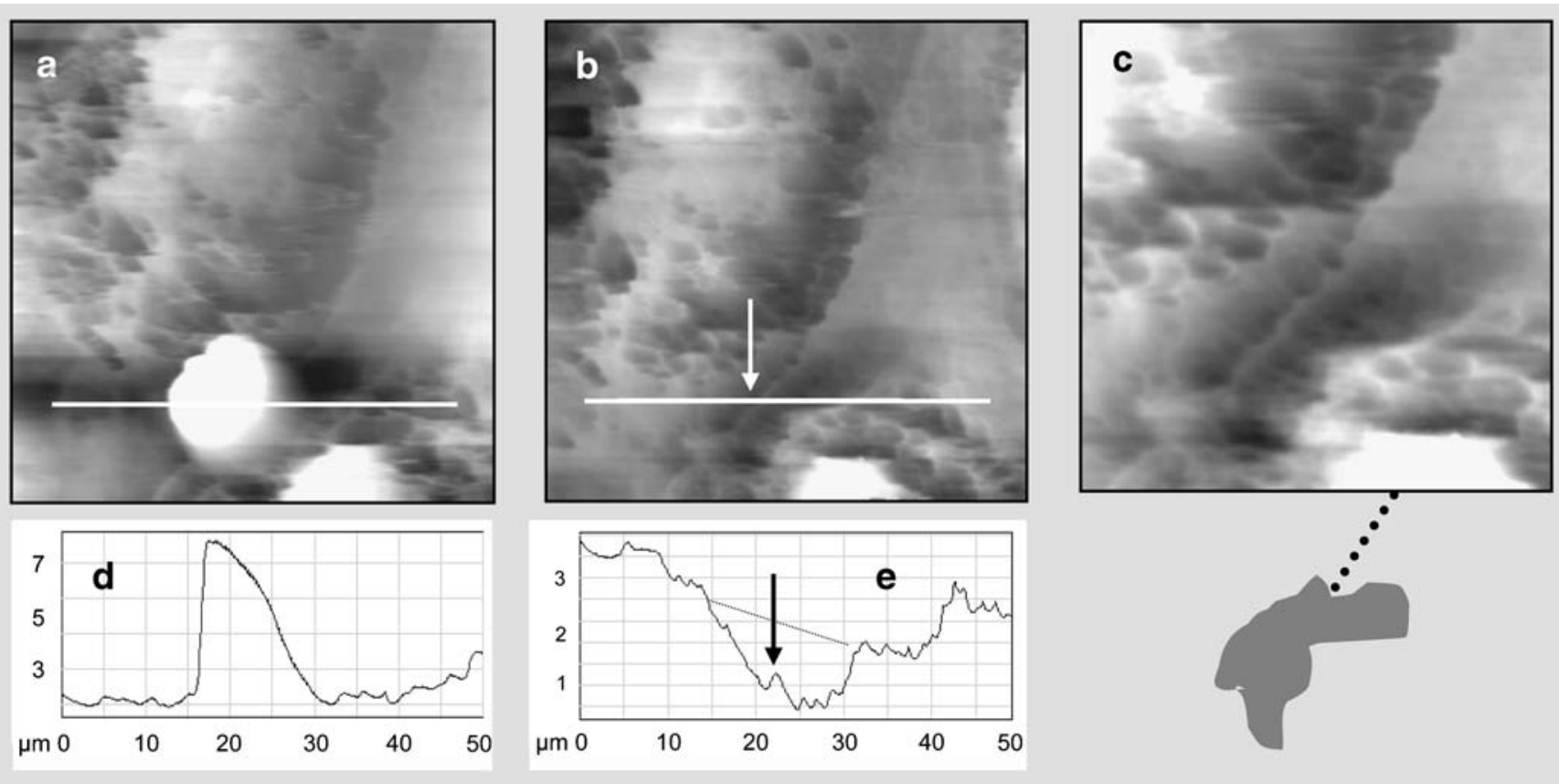

Fig. 6 Segmented docking structure in human cells. A HUVEC/blood neutrophil cell sample underwent the nanomanipulation procedure as depicted in Fig. 3. a AFM image before and $\mathbf{b}$ after the removal of the leukocyte; c zoom into the docking region is illustrated by the sketch

IL $\beta$ stimulation and adherent monocytes. The authors found a softening of the endothelium but, at the same time, could not observe an alteration of cell-to-cell distances as measured by electrical impedance spectroscopy [10].

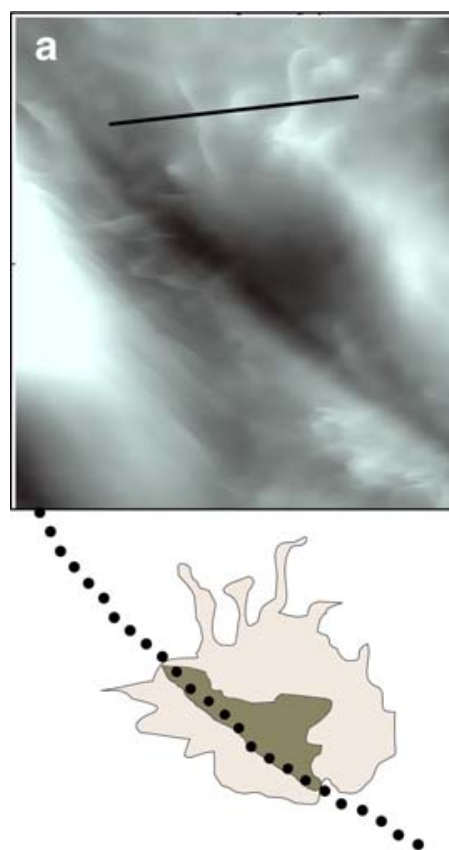

Fig. 7 Filopodial protrusions around a CLDS. Samples were prepared as for Fig. 5a. AFM contact image of the CLDS in the endothelial cell; in the sketch below, the interface area is shaded brightly, the actual cup darkly, and the cell junction is denoted as a dotted line. b Zoom into thereunder: the interface region is shaded brightly, the cup region in dark and the cell junction is given as a dotted line. In $\mathbf{d}$ and $\mathbf{e}$, the corresponding height profiles are given with arrows denoting the ridge at endothelial cell junction

Our analysis of the mechanical properties of the endothelial cell surface so far does not point to a local actin depolymerization at the site of firm adhesion. More experiments have to be performed, and the protocol has to
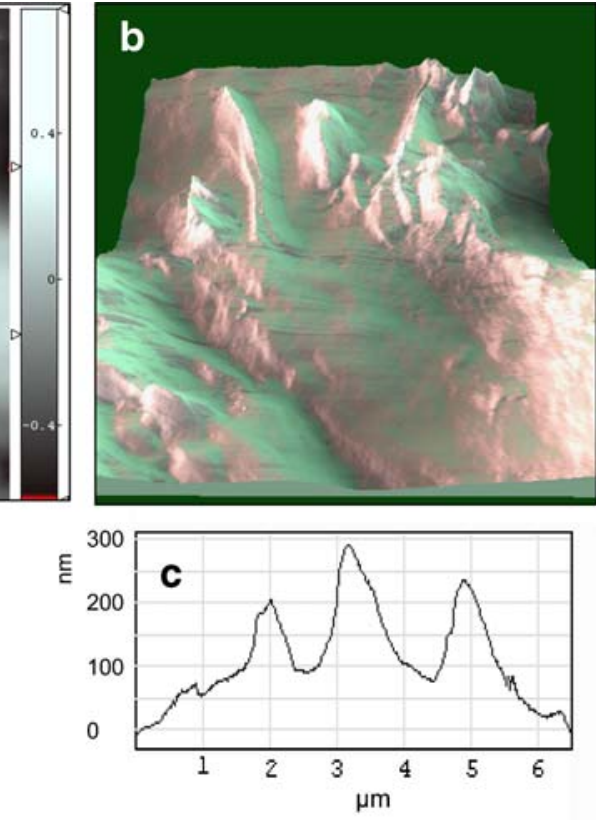

the rim with the filopodia at the border of the contact region in threedimensional presentation. c Height profile of the filopodia along the white line in a 


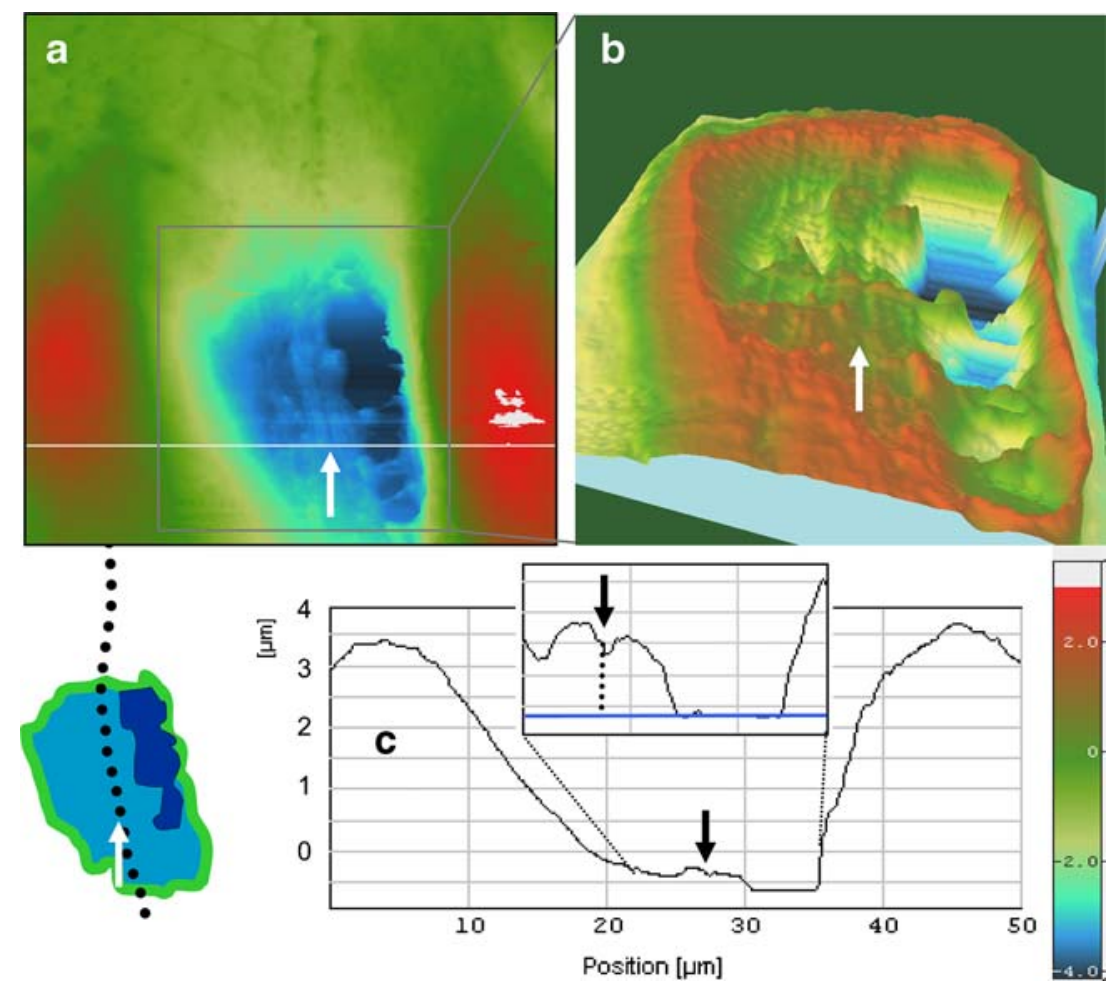

Fig. 8 A transmigratory channel close to an endothelial cell junction. The sample was prepared as as in Fig. 6. a AFM contact image of a docking structure that already perforated the endothelial cell, as it reached the glass bottom of the culture dish, here depicted in dark blue (note the color scale, lower right). b Zoom into the channel region of a in three-dimensional demonstration; the sketch at the lower left depicts

be optimized to obtain data from non-fixed specimens. Then, also small differences in local sample elasticity should become detectable, eventually giving a hint to cytoskeletal rearrangements preceding CLDS formation.

Physiological relevance Altogether, we have found six CLDSs (three in each cell system), five of which are shown in this paper. All lie close to and four of them even across an endothelial junction. In most cases, the border appears to be intact, with junctional f-actin bundles forming a ridge within the invagination, hence segmenting the CLDS into two parts (Fig. 6, arrow). So far, transmigrating leukocytes in direct vicinity of endothelial junctions have been carefully counted as cells potentially migrating through a paracellular route to not overestimate transcellular events [8]. Our results show that transmigration of leukocytes is indeed possible in a transcellular manner even in close proximity of the paracellular cleft. Of course, the low number of CLDS that we could observe does not allow statistically relevant statements about the percentage of transcellular versus paracellular migrating cells.

Not much is known about a potential guidance mechanism for the transcellular migration route. ICAM-1 and the interface region (bright), the channel (dark), and the cell border (dotted line). c From the height profile along the white line in a, the two cortical actin fiber bundles flanking the cell junction can be made out. The neighboring flat horizontal line represents the glass bottom (inset)

caveolae have been suggested to be involved in this process $[13,31]$. One possible mechanism would be that endothelial cells engulf leukocytes in a phagocytic-like way via the endothelial filopodia that have been described in an ex vivo study on the blood-brain barrier [29]. This would create a situation where the filopodia are embracing the leukocyte and already fuse above the leukocyte before it moves through the endothelial cells. We found no images in our study that would support this model. Instead, we found that the transmigratory channel could be imaged spanning through the whole endothelial cell from the apical to the basal side. In fact, the fluorescence microscopy images presented by Carman and Springer [6] also showed that the leukocyte already started spreading underneath the endothelial cell layer while the rear end of it was still reaching the lumen. We speculate that during the transcellular migration process, the apical endothelial membrane forming the bottom of the CLDS touches the basal endothelial membrane at a final step of transmigration. This then will probably force both membranes to fuse, giving rise to an opening that allows the leukocyte to move through the endothelial cell.

It is conceivable that such a process would occur at sites where the endothelial cell is very thin. Such sites most likely are found at the rim of a cell close to endothelial cell 
contacts. In vitro, the lamellipodium of endothelial cells often is as thin as $200 \mathrm{~nm}$. These geometrical conditions might be the reason why transcellular migrating leukocytes are often found close to endothelial cell contacts. In conclusion, AFM nanomanipulation uniquely makes the leukocyte-endothelial contact site accessible and adds novel perspectives to the study of the diapedesis process.

Acknowledgment We would like to thank Birgit Kempe and Marianne Wilhelmi for the excellent technical assistance. This work was supported by the fund "Innovative Medical Research" of the University of Munster Medical School, RI 220506.

\section{References}

1. Adamson P, Etienne S, Couraud PO, Calder V, Greenwood J (1999) Lymphocyte migration through brain endothelial cell monolayers involves signaling through endothelial ICAM-1 via a rho-dependent pathway. J Immunol 162:2964-2973

2. Allingham MJ, van Buul JD, Burridge K (2007) ICAM-1mediated, Src- and Pyk2-dependent vascular endothelial cadherin tyrosine phosphorylation is required for leukocyte transendothelial migration. J Immunol 179:4053-4064

3. Barreiro O, Yanez-Mo M, Serrador JM, Montoya MC, VicenteManzanares M, Tejedor R, Furthmayr H, Sanchez-Madrid F (2002) Dynamic interaction of VCAM-1 and ICAM-1 with moesin and ezrin in a novel endothelial docking structure for adherent leukocytes. J Cell Biol 157:1233-1245

4. Bixel MG, Petri B, Khandoga AG, Khandoga A, Wolburg-Buchholz K, Wolburg H, Marz S, Krombach F, Vestweber D (2007) A CD99related antigen on endothelial cells mediates neutrophil but not lymphocyte extravasation in vivo. Blood 109:5327-5336

5. Carman CV, Jun CD, Salas A, Springer TA (2003) Endothelial cells proactively form microvilli-like membrane projections upon intercellular adhesion molecule 1 engagement of leukocyte LFA1. J Immunol 171:6135-6144

6. Carman CV Springer TA (2004) A transmigratory cup in leukocyte diapedesis both through individual vascular endothelial cells and between them. J Cell Biol 167:377-388

7. Discher DE, Mohandas N, Evans EA (1994) Molecular maps of red cell deformation: hidden elasticity and in situ connectivity. Science 266:1032-1035

8. Eibl RH Moy VT (2005) Atomic force microscopy measurements of protein-ligand interactions on living cells. Methods Mol Biol 305:439-450

9. Feng D, Nagy JA, Pyne K, Dvorak HF, Dvorak AM (1998) Neutrophils emigrate from venules by a transendothelial cell pathway in response to FMLP. J Exp Med 187:903-915

10. Kataoka N, Iwaki K, Hashimoto K, Mochizuki S, Ogasawara Y, Sato M, Tsujioka K, Kajiya F (2002) Measurements of endothelial cell-to-cell and cell-to-substrate gaps and micromechanical properties of endothelial cells during monocyte adhesion. Proc Natl Acad Sci U S A 99:15638-15643

11. Langer F, Morys-Wortmann C, Kusters B, Storck J (1999) Endothelial protease-activated receptor-2 induces tissue factor expression and von Willebrand factor release. Br J Haematol 105:542-550

12. Michel CC Curry FE (1999) Microvascular permeability. Physiol Rev 79:703-761

13. Millan J, Hewlett L, Glyn M, Toomre D, Clark P, Ridley AJ (2006) Lymphocyte transcellular migration occurs through recruitment of endothelial ICAM-1 to caveola- and F-actin-rich domains. Nat Cell Biol 8:113-123
14. Muller WA (2003) Leukocyte-endothelial cell interactions in leukocyte transmigration and the inflammatory response. Trends Immunol 24:327-334

15. Oberleithner $H$ (2007) Is the vascular endothelium under the control of aldosterone? Facts and hypothesis. Pflugers Arch 454:187-193

16. Oberleithner H, Riethmuller C, Ludwig T, Shahin V, Stock C, Schwab A, Hausberg M, Kusche K, Schillers H (2006) Differential action of steroid hormones on human endothelium. J Cell Sci 119:1926-1932

17. Petri B Bixel MG (2006) Molecular events during leukocyte diapedesis. FEBS J 273:4399-4407

18. Reiss Y, Hoch G, Deutsch U, Engelhardt B (1998) T cell interaction with ICAM-1-deficient endothelium in vitro: essential role for ICAM-1 and ICAM-2 in transendothelial migration of T cells. Eur J Immunol 28:3086-3099

19. Riethmuller C, Jungmann P, Wegener J, Oberleithner H (2006) Bradykinin shifts endothelial fluid passage from para- to transcellular routes. Pflugers Arch 453:157-165

20. Rotsch C, Braet F, Wisse E, Radmacher M (1997) AFM imaging and elasticity measurements on living rat liver macrophages. Cell Biol Int 21:685-696

21. Sato M, Nagayama K, Kataoka N, Sasaki M, Hane K (2000) Local mechanical properties measured by atomic force microscopy for cultured bovine endothelial cells exposed to shear stress. J Biomech 33:127-135

22. Schneider SW, Pagel P, Rotsch C, Danker T, Oberleithner H, Radmacher M, Schwab A (2000) Volume dynamics in migrating epithelial cells measured with atomic force microscopy. Pflugers Arch 439:297-303

23. Takei $F$ (1985) Inhibition of mixed lymphocyte response by a rat monoclonal antibody to a novel murine lymphocyte activation antigen (MALA-2). J Immunol 134:1403-1407

24. van Buul JD, Allingham MJ, Samson T, Meller J, Boulter E, Garcia-Mata R, Burridge K (2007) RhoG regulates endothelial apical cup assembly downstream from ICAM1 engagement and is involved in leukocyte trans-endothelial migration. J Cell Biol 178:1279-1293

25. Vestweber D (2007) Adhesion and signaling molecules controlling the transmigration of leukocytes through endothelium. Immunol Rev 218:178-196

26. Vestweber D (2003) Lymphocyte trafficking through blood and lymphatic vessels: more than just selectins, chemokines and integrins. Eur J Immunol 33:1361-1364

27. Wang S, Dangerfield JP, Young RE, Nourshargh S (2005) PECAM-1, alpha6 integrins and neutrophil elastase cooperate in mediating neutrophil transmigration. J Cell Sci 118:2067-2076

28. Wojcikiewicz EP, Abdulreda MH, Zhang X, Moy VT (2006) Force spectroscopy of LFA-1 and its ligands, ICAM-1 and ICAM2. Biomacromolecules 7:3188-3195

29. Wolburg H, Wolburg-Buchholz K, Engelhardt B (2005) Diapedesis of mononuclear cells across cerebral venules during experimental autoimmune encephalomyelitis leaves tight junctions intact. Acta Neuropathol (Berl) 109:181-190

30. Wolburg K, Gerhardt H, Schulz M, Wolburg H, Engelhardt B (1999) Ultrastructural localization of adhesion molecules in the healthy and inflamed choroid plexus of the mouse. Cell Tissue Res 296:259-269

31. Yang L, Froio RM, Sciuto TE, Dvorak AM, Alon R, Luscinskas FW (2005) ICAM-1 regulates neutrophil adhesion and transcellular migration of TNF-alpha-activated vascular endothelium under flow. Blood 106:584-592

32. Zhang X, Chen A, De Leon D, Li H, Noiri E, Moy VT, Goligorsky MS (2004) Atomic force microscopy measurement of leukocyte-endothelial interaction. Am J Physiol Heart Circ Physiol 286:H359-H367 